\title{
Extremal problems for game domination number
}

\author{
William B. Kinnersley*, Douglas B. West ${ }^{\dagger}$ Reza Zamani ${ }^{\ddagger}$
}

August 22, 2013

\begin{abstract}
In the domination game on a graph $G$, two players called Dominator and Staller alternately select vertices of $G$. Each vertex chosen must strictly increase the number of vertices dominated; the game ends when the chosen set becomes a dominating set of $G$. Dominator aims to minimize the size of the resulting dominating set, while Staller aims to maximize it. When both players play optimally, the size of the dominating set produced is the game domination number of $G$, denoted by $\gamma_{g}(G)$ when Dominator plays first and by $\gamma_{g}^{\prime}(G)$ when Staller plays first.

We prove that $\gamma_{g}(G) \leq 7 n / 11$ when $G$ is an isolate-free $n$-vertex forest and that $\gamma_{g}(G) \leq\lceil 7 n / 10\rceil$ for any isolate-free $n$-vertex graph. In both cases we conjecture that $\gamma_{g}(G) \leq 3 n / 5$ and prove it when $G$ is a forest of nontrivial caterpillars. We also resolve conjectures of Brešar, Klavžar, and Rall by showing that always $\gamma_{g}^{\prime}(G) \leq \gamma_{g}(G)+1$, that for $k \geq 2$ there are graphs $G$ satisfying $\gamma_{g}(G)=2 k$ and $\gamma_{g}^{\prime}(G)=2 k-1$, and that $\gamma_{g}^{\prime}(G) \geq \gamma_{g}(G)$ when $G$ is a forest. Our results follow from fundamental lemmas about the domination game that simplify its analysis and may be useful in future research.
\end{abstract}

\section{Introduction}

Recent years have seen growing interest in competitive optimization on graphs. Here two agents with conflicting goals together construct some structure on an underlying graph; often, these problems draw inspiration from classical graph parameters.

For example, the celebrated game chromatic number arises from graph coloring. Alice and Bob take turns coloring vertices of a given graph from a given palette of colors, without giving adjacent vertices the same color. Alice seeks a proper coloring of the full graph, while Bob aims to prevent this. The minimum number of colors needed for Alice to have a winning

\footnotetext{
*Mathematics Department, University of Illinois, Urbana IL 61801, wkinners@gmail.com. Research supported by National Science Foundation grant DMS 08-38434 "EMSW21-MCTP: Research Experience for Graduate Students".

${ }^{\dagger}$ Mathematics Department, University of Illinois, Urbana IL 61801, west@math.uiuc.edu. Research supported by the National Security Agency under Award No. H98230-09-1-0363.

${ }_{\ddagger}^{\ddagger}$ Computer Science Department, University of Illinois, Urbana IL 61801, rezazamaninasab@gmail.com.
} 
strategy is the game chromatic number of $G$. Introduced by Gardner [8], this parameter has received much attention (see $[5,10,11,15]$ ). Competitive optimization parameters have also arisen from game variants of list coloring $([16,13])$ and graph saturation $([7])$.

A dominating set in a graph $G$ is a subset $S$ of $V(G)$ such that every vertex outside $S$ has a neighbor in $S$ (vertices dominate themselves). The domination number $\gamma(G)$ is the minimum size of a dominating set. Nearly a thousand papers and several books have been written on variations of domination; see [9] for a summary of the basic material.

Brešar, Klavžar, and Rall [2] introduced a game variant of graph domination, which they attributed to Henning. In the domination game on a graph $G$, two players called Dominator and Staller take turns choosing vertices of $G$. Each added vertex must dominate at least one vertex not dominated by previously chosen vertices. The game ends when the chosen set becomes a dominating set. Dominator wants to minimize the size of the final dominating set, while Staller wants to maximize it, prolonging the game. The game domination number of $G$ is the size of the resulting set when both players play optimally, denoted by $\gamma_{g}(G)$ in the variant where Dominator plays first and by $\gamma_{g}^{\prime}(G)$ when Staller plays first. (This parameter differs from the parameter called "game domination number" by Alon, Balogh, Bollobás, and Szabó [1] and studied also in [6].)

Section 2 develops ideas and lemmas that simplify reasoning about the domination game. Lemma 2.1 formalizes the intuition that starting the game with some vertices already dominated cannot lengthen the game; this resolves a question posed in [2]. Next, we introduce a variant of the game where Staller may skip moves. We denote the number of vertices played in this variant under optimal play by $\hat{\gamma}_{g}$ or $\hat{\gamma}_{g}^{\prime}$, depending on who moves first. Strengthening Staller by providing this option yields a game that is easier to analyze than the original game and provides upper bounds. Lemma 2.4 says that if $G=G_{1} \cup G_{2}$, then $\hat{\gamma}_{g}(G) \leq \hat{\gamma}_{g}\left(G_{1}\right)+\hat{\gamma}_{g}^{\prime}\left(G_{2}\right)$, and hence also $\gamma_{g}(G) \leq \hat{\gamma}_{g}\left(G_{1}\right)+\hat{\gamma}_{g}^{\prime}\left(G_{2}\right)$.

Section 3 provides two general upper bounds on $\gamma_{g}$. Theorem 3.1 strengthens the bound $\gamma_{g}(G) \leq 2 \gamma(G)-1$ observed in [2]. Next, we use Lemma 2.4 and Theorem 3.1 to prove that $\gamma_{g}(G) \leq\lceil 7 n / 10\rceil$ for every $n$-vertex graph $G$ without isolated vertices.

Section 4 continues the work begun in [2] on determining which pairs $\left(k, k^{\prime}\right)$ are realizable as $\left(\gamma_{g}(G), \gamma_{g}^{\prime}(G)\right)$ for some graph $G$. In [2] it was shown that all realizable pairs have the form $(k, k-1),(k, k),(k, k+1)$, or $(k, k+2)$, that all pairs of the form $(k, k),(k, k+1)$, or $(2 k+1,2 k)$ are realizable, and that $(2,1)$ is not. They conjectured that $(k, k+2)$ and $(2 k, 2 k-1)$ are never realizable. We confirm that $(k, k+2)$ is not realizable, but $(2 k, 2 k-1)$ is realizable when $k \geq 2$. We also prove a conjecture from [3] by showing that $\gamma_{g}(G) \leq \gamma_{g}^{\prime}(G)$ when $G$ is a forest. Finally, we show that equality in the bound $\gamma_{g}(G) \leq 2 \gamma(G)-1$ can be achieved by $n$-vertex graphs with both domination number and connectivity as high as $\lfloor\sqrt{n}\rfloor$.

Section 5 studies the maximum value of $\gamma_{g}$ over $n$-vertex forests. Since $\gamma_{g}\left(\bar{K}_{n}\right)=n$, we consider only isolate-free forests (i.e., those without isolated vertices). Theorem 5.5 states that $\gamma_{g}(G) \leq 3 n / 5$ when each component of $G$ is a nontrivial caterpillar (a caterpillar is a 
tree in which the non-leaf vertices form a path, and a graph is nontrivial if it has at least one edge). This bound is sharp, and we conjecture that it holds for all isolate-free forests. Theorem 5.6 is a partial result toward this conjecture, giving a weaker upper bound of $7 n / 11$. We conclude with several interesting open questions.

Throughout the paper, we write $N(v)$ for the neighborhood of a vertex $v$ in a graph $G$; the closed neighborhood is $N(v) \cup\{v\}$, denoted $N[v]$. During a domination game on a graph $G$, we call a vertex $v$ dominated if some vertex within $N[v]$ has been selected.

To simplify discussion, we adopt terminology from games. A turn is the phase of the game consisting of one player's action to select a vertex or to pass. When a player completes that action, we say that he moves; when that action is selecting a vertex $v$, we refer to $v$

as the "move" and say that he "plays" $v$. When Dominator moves first, the game is the Dominator-start game; when Staller moves first, it is the Staller-start game.

\section{The Segmentation Lemma}

Our goal in this section is a lemma that will be used throughout the paper. We begin by resolving a conjecture of Brešar, Klavžar, and Rall [2].

A partially-dominated graph is a graph together with a declaration that some vertices are already considered dominated (or, equivalently, do not "need" to be dominated). When $G$ is a partially-dominated graph, we denote the number of turns remaining in the game under optimal play by $\gamma_{g}(G)$ and $\gamma_{g}^{\prime}(G)$, depending on who moves first.

Problem 1 in [2] asked whether having more vertices dominated could ever hurt Dominator. In particular, if the set of "pre-dominated" vertices in a graph is enlarged, can the game domination number be larger? Our first lemma shows that it cannot, formalizing the natural intuition. We invoke it frequently throughout the paper, often without explicit mention. We use the name for it suggested in [3].

Lemma 2.1 (Continuation Principle). Let $G$ be a graph, and fix $A, B \subseteq V(G)$. Let $G_{A}$ and $G_{B}$ be the partially-dominated graphs arising from $G$ with $A$ dominated and with $B$ dominated, respectively. If $B \subseteq A$, then $\gamma_{g}\left(G_{A}\right) \leq \gamma_{g}\left(G_{B}\right)$ and $\gamma_{g}^{\prime}\left(G_{A}\right) \leq \gamma_{g}^{\prime}\left(G_{B}\right)$.

Proof. Dominator simultaneously plays Game A on $G_{A}$ and Game B on $G_{B}$. Game A is the "real" game that both players play, while Game B is imagined by Dominator. Dominator ensures that Game A finishes no later than Game B by maintaining the following invariant: every dominated vertex in Game B is also dominated in Game A. By assumption, this holds at the beginning. We consider the moves of Staller and Dominator separately.

On each turn, Staller plays in Game A. By the invariant, Staller's move in Game A is also available in Game B. Dominator imagines that Staller makes the same move in Game $\mathrm{B}$; the invariant is maintained. 
On each turn, Dominator plays in Game B using an optimal strategy for that game. If Dominator's move $v$ in Game B is a valid move in Game A, then he plays it in Game A, maintaining the invariant. Otherwise, every newly-dominated vertex in Game B is already dominated in Game A, and the invariant holds regardless of Dominator's move; he plays any undominated vertex in Game A.

By the invariant, Game A finishes no later than Game B. Since Dominator played optimally on Game B, the number of turns there was at most $\gamma_{g}\left(G_{B}\right)$ if he moved first and at most $\gamma_{g}^{\prime}\left(G_{B}\right)$ if Staller moved first. Thus $\gamma_{g}\left(G_{A}\right) \leq \gamma_{g}\left(G_{B}\right)$ and $\gamma_{g}^{\prime}\left(G_{A}\right) \leq \gamma_{g}^{\prime}\left(G_{B}\right)$.

Remark 2.2. By the Continuation Principle, it never helps Dominator to make moves that dominate no new vertices. Therefore, we need not restrict Dominator's moves; henceforth we permit him to play any vertex, whether or not it dominates any additional vertices. (Indeed we may even allow Dominator to select vertices that have already been played, so long as we adopt the convention that these "redundant" vertices contribute with multiplicity toward the size of the final dominating set.)

Strengthening Dominator in this way does not change the length of the game (under optimal play), but it does ensure that when presenting strategies we need not check that Dominator's moves are valid. (Alternatively, the reader may imagine that Dominator, when directed to play an "illegal" move, instead plays an arbitrary legal move.)

It can also help to consider variants of the domination game in which Staller is stronger. In [2], the authors briefly explored a variant in which Staller can pass once during the game; more generally, we may allow Staller to pass on any turn.

Definition 2.3. The Staller-pass game is the variant of the domination game in which, on each turn, Staller may pass instead of playing a vertex. Denote the size of the final dominating set in the Staller-pass game on $G$, under optimal play, by $\hat{\gamma}_{g}(G)$ if Dominator moves first and by $\hat{\gamma}_{g}^{\prime}(G)$ if Staller moves first. (Turns on which Staller passes do not count toward the size of the final dominating set.)

Since Staller has additional options in the Staller-pass game and Dominator does not, $\hat{\gamma}_{g}(G) \geq \gamma_{g}(G)$ and $\hat{\gamma}_{g}^{\prime}(G) \geq \gamma_{g}^{\prime}(G)$ for any graph $G$. Moreover, since Staller may pass initially, always $\hat{\gamma}_{g}(G) \leq \hat{\gamma}_{g}^{\prime}(G)$. This property contrasts with the usual game, where sometimes $\gamma_{g}(G)>\gamma_{g}^{\prime}(G)$. Thus the Staller-pass game is "better-behaved" than the usual game; playing first cannot be a disadvantage to a Staller who can pass.

The Continuation Principle applies also to the Staller-pass game; the same proof works. We use this observation to prove an important lemma. The union $G$ of partially-dominated graphs $G_{1}$ and $G_{2}$ is obtained by letting the graph $G$ be the graph union $G_{1} \cup G_{2}$ and letting the set of dominated vertices in $G$ be the union of the dominated sets in $G_{1}$ and $G_{2}$.

We now present the Segmentation Lemma, which facilitates inductive proofs of upper bounds on $\gamma_{g}$. When we express a graph $G$ as the union of "nice" graphs $G_{1}$ and $G_{2}$, the 
Segmentation Lemma provides a bound on the length of the Staller-pass game on $G$ in terms of the lengths of the Staller-pass games on $G_{1}$ and $G_{2}$. Since $\gamma_{g}(G) \leq \hat{\gamma}_{g}(G)$ and $\gamma_{g}^{\prime}(G) \leq \hat{\gamma}_{g}^{\prime}(G)$, these bounds apply also to the original game.

Lemma 2.4 (Segmentation Lemma). Let $G_{1}$ and $G_{2}$ be partially-dominated graphs. If $G=$ $G_{1} \cup G_{2}$, then $\hat{\gamma}_{g}(G) \leq \hat{\gamma}_{g}\left(G_{1}\right)+\hat{\gamma}_{g}^{\prime}\left(G_{2}\right)$ and $\hat{\gamma}_{g}^{\prime}(G) \leq \hat{\gamma}_{g}^{\prime}\left(G_{1}\right)+\hat{\gamma}_{g}^{\prime}\left(G_{2}\right)$.

Proof. Dominator constructs two auxiliary games, one on $G_{1}$ and one on $G_{2}$, which we call sub-games. Throughout the course of the game on $G$, Dominator interprets each of Staller's moves as a move in one of the sub-games, then uses an optimal strategy for that sub-game to determine his own next move on $G$. In doing this, Dominator ensures that, at all times, every vertex dominated in one of the sub-games is also dominated in $G$.

If Dominator moves first, then he plays in the sub-game on $G_{1}$ according to some optimal strategy for that game and makes the same move on $G$. For the remainder of the game Dominator plays reactively, responding to Staller's moves. Hence the strategy also applies to the Staller-start game.

If Staller passes, then Dominator responds in an arbitrary sub-game, playing as if Staller had passed in that sub-game. If instead Staller plays some vertex $v$, then Dominator finds some vertex $w$ that was newly dominated by this move. If $v w \in E\left(G_{1}\right)$, then Staller's last move would have been valid in the sub-game on $G_{1}$, so Dominator views it as a move in that sub-game and responds there. Otherwise, Staller's last move would have been valid in the sub-game on $G_{2}$, so Dominator views it as a move in the sub-game on $G_{2}$ and responds there. At some point it may happen that Staller finishes one of the sub-games, in which case Dominator acts as if Staller had passed in the other sub-game and responds there. In each case, Dominator makes the same move on $G$ as in the sub-game. (Note that vertices dominated in $G$ need not be dominated in both sub-games, so a move that dominates new vertices in a sub-game need not dominate new vertices in $G$. By Remark 2.2, we may allow Dominator to make such a move on $G$.)

Since Dominator played first in the sub-game on $G_{1}$, at most $\hat{\gamma}_{g}\left(G_{1}\right)$ moves are played there. Also, at most $\hat{\gamma}_{g}^{\prime}\left(G_{2}\right)$ moves are played in the sub-game on $G_{2}$. Since every vertex dominated in a sub-game is also dominated in $G$, the game must end by the time both sub-games have been completed. Thus $\hat{\gamma}_{g}(G) \leq \hat{\gamma}_{g}\left(G_{1}\right)+\hat{\gamma}_{g}^{\prime}\left(G_{2}\right)$. The same strategy yields the claimed bound on $\hat{\gamma}_{g}^{\prime}(G)$; in this case, Staller starts in both sub-games.

\section{Bounds for $n$-vertex graphs}

For our general bound, instead of bounding $\gamma_{g}(G)$ directly, we bound $\hat{\gamma}_{g}^{\prime}(G)$, giving a stronger result. When $m=\gamma(G)$, the bound usually improves the bound $\gamma_{g}(G) \leq 2 \gamma(G)-1$ from [2]. 
Theorem 3.1. Let $G$ be an n-vertex graph. If $m$ and $k$ are positive integers such that $\gamma(G) \leq m \leq n \leq m k$, then $\hat{\gamma}_{g}^{\prime}(G) \leq\left\lceil 2 m\left(1-2^{-k}\right)\right\rceil$.

Proof. Staller starts and can pass. Dominator fixes a dominating set $S$ of size $m$ in $G$. On each turn, Dominator plays a vertex of $S$ that dominates the most new vertices. Suppose first that $m=c 2^{k-1}$ for some integer $c$; we show later how to deal with arbitrary $m$.

For $0 \leq i \leq k-1$, we show by induction on $i$ that at most $m 2^{-i}(k-i)$ vertices in $G$ remain undominated when $2 m\left(1-2^{-i}\right)$ vertices have been played. Initially, $i=0$.

Suppose that $2 m\left(1-2^{-i}\right)$ vertices have been played and at most $m 2^{-i}(k-i)$ vertices remain undominated. Note that $2 m\left(1-2^{-(i+1)}\right)-2 m\left(1-2^{-i}\right)=2 m 2^{-(i+1)}$. Consider the state of the game after $2 m 2^{-(i+1)}$ more vertices have been played. If every vertex in $S$ now has fewer than $k-i$ undominated vertices in its closed neighborhood, then since Dominator has played at least $m\left(1-2^{-(i+1)}\right)$ vertices in $S$, at most $m 2^{-(i+1)}$ vertices in $S$ have not been played, and at most $m 2^{-(i+1)}(k-i-1)$ vertices remain undominated, as desired.

Otherwise, at least $k-i$ new vertices were dominated by each of Dominator's last $m 2^{-(i+1)}$ moves. Moreover, each of the other $m 2^{-(i+1)}$ vertices played among the last $2 m 2^{-(i+1)}$ (whether played by Staller or Dominator) dominated at least one new vertex. In total, the last $2 m 2^{-(i+1)}$ vertices played dominated at least $m 2^{-(i+1)}(k-i+1)$ new vertices, so the number of undominated vertices remaining is at most $m 2^{-i}(k-i)-m 2^{-(i+1)}(k-i+1)$, which simplifies to $m 2^{-(i+1)}(k-i-1)$. This completes the induction step.

With $i=k-1$, at most $m 2^{-(k-1)}$ vertices remain undominated after $2 m\left(1-2^{-(k-1)}\right)$ vertices are played. Thus at most $m 2^{-(k-1)}$ more vertices will be played. Hence the size of the final dominating set is at most $2 m\left(1-2^{-(k-1)}\right)+m 2^{-(k-1)}$, which equals $2 m\left(1-2^{-k}\right)$.

When $m$ is not a multiple of $2^{k-1}$, let $m=c 2^{k-1}+r$ for integers $c$ and $r$ with $0 \leq r<2^{k-1}$. After the first $2 r$ turns, Dominator has played $r$ of the $m$ vertices in $S$, greedily, so at most $n\left(1-\frac{r}{m}\right)$ vertices remain undominated. Since $n \leq m k$, we have $n\left(1-\frac{r}{m}\right) \leq k(m-r)=k c 2^{k-1}$. Applying the same analysis used above shows that at most $2\left(c 2^{k-1}\right)-c$ more vertices are played during the game, so $\hat{\gamma}_{g}^{\prime}(G) \leq 2 r+2\left(c 2^{k-1}\right)-c=\left\lceil 2 m\left(1-2^{-k}\right)\right\rceil$.

The Segmentation Lemma and Theorem 3.1 together yield an upper bound for isolate-free $n$-vertex graphs. We also use a well-known theorem of Blank [4]:

Theorem 3.2 (Blank). If $G$ is an n-vertex graph with minimum degree at least 2, then $\gamma(G) \leq 2 n / 5$, unless $G$ is in the set $\mathcal{F}$ of seven graphs drawn below:
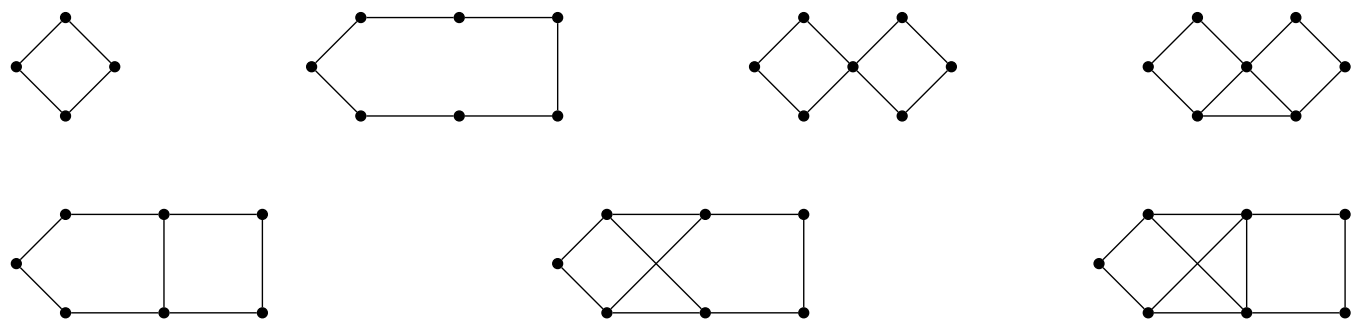
In the proof below, and often throughout the paper, to facilitate induction we discard vertices of a partially-dominated graph that no longer affect the domination game.

Definition 3.3. In a partially-dominated graph $G$, a vertex $v$ in $G$ is saturated if all of its closed neighborhood is dominated. The residual graph of $G$ is the partially-dominated graph formed from $G$ by removing all saturated vertices and all edges joining dominated vertices.

Removing saturated vertices from a partially-dominated graph does not change its game domination number, since Staller cannot play saturated vertices and Dominator has no reason to. Similarly, removing an edge joining two dominated vertices does not affect the game.

Theorem 3.4. If $G$ is an isolate-free n-vertex graph, then $\gamma_{g}(G) \leq\left\lceil\frac{7 n}{10}\right\rceil$.

Proof. Using induction on $n$, we prove the stronger statement $\hat{\gamma}_{g}(G) \leq\lceil 7 n / 10\rceil$. To facilitate induction, we prove also that $\hat{\gamma}_{g}^{\prime}(G) \leq\lceil(7 n+3) / 10\rceil$, and we further generalize both claims by allowing $G$ to be partially-dominated. Both claims are true by inspection when $n \leq 3$.

Suppose that Staller has the first turn. If the optimal first move is a pass, then $\hat{\gamma}_{g}^{\prime}(G)=$ $\hat{\gamma}_{g}(G)$, and it suffices to prove $\hat{\gamma}_{g}^{\prime}(G)=\hat{\gamma}_{g}(G) \leq\left\lceil\frac{7 n}{10}\right\rceil$. If the optimal first move is a vertex $v$, then $v$ becomes saturated. Letting $G^{\prime}$ be the resulting residual graph,

$$
\hat{\gamma}_{g}^{\prime}(G)=1+\hat{\gamma}_{g}\left(G^{\prime}\right) \leq 1+\left\lceil\frac{7(n-1)}{10}\right\rceil=\left\lceil\frac{7 n+3}{10}\right\rceil .
$$

Now suppose that Dominator plays first. If $G$ has a vertex of degree 1, then let $v$ be such a vertex, and let $u$ be its neighbor. Dominator plays $u$, saturating both $u$ and $v$. Let $G^{\prime}$ be the resulting residual graph. Since $G^{\prime}$ has at most $n-2$ vertices,

$$
\hat{\gamma}_{g}(G) \leq 1+\hat{\gamma}_{g}^{\prime}\left(G^{\prime}\right) \leq 1+\left\lceil\frac{7(n-2)+3}{10}\right\rceil=\left\lceil\frac{7 n-1}{10}\right\rceil .
$$

Hence we may assume $\delta(G) \geq 2$. If $G$ has a component $C$ isomorphic to $C_{4}$, then the Segmentation Lemma yields

$$
\hat{\gamma}_{g}(G) \leq \hat{\gamma}_{g}(C)+\hat{\gamma}_{g}^{\prime}(G-V(C)) \leq 2+\left\lceil\frac{7(n-4)+3}{10}\right\rceil=\left\lceil\frac{7 n-5}{10}\right\rceil .
$$

If $G$ has a component $C$ isomorphic to another graph in the set $\mathcal{F}$ of Theorem 3.2 , then $\hat{\gamma}_{g}(C) \leq 3$ by inspection. This time the Segmentation Lemma yields

$$
\hat{\gamma}_{g}(G) \leq \hat{\gamma}_{g}(C)+\hat{\gamma}_{g}^{\prime}(G-V(C)) \leq 3+\left\lceil\frac{7(n-7)+3}{10}\right\rceil=\left\lceil\frac{7 n-16}{10}\right\rceil .
$$

Finally, if no component of $G$ lies in $\mathcal{F}$, then $\gamma(G) \leq 2 n / 5$ by Theorem 3.2. Applying Theorem 3.1 with $m=2 n / 5$ and $k=3$ yields

$$
\hat{\gamma}_{g}^{\prime}(G) \leq\left\lceil 2 \cdot \frac{2 n}{5} \cdot\left(1-\frac{1}{8}\right)\right\rceil=\left\lceil\frac{7 n}{10}\right\rceil .
$$

We have no sharpness examples for Theorem 3.4. Indeed, we believe that the bound can be improved to $3 n / 5$ (see Conjecture 6.2). 


\section{Realizability}

A pair $\left(k, k^{\prime}\right)$ is realizable if $\gamma_{g}(G)=k$ and $\gamma_{g}^{\prime}(G)=k^{\prime}$ for some graph $G$. The study of realizable pairs was a major focus of [2], where the authors showed that all realizable pairs have one of the forms $(k, k-1),(k, k),(k, k+1)$, and $(k, k+2)$. They also showed that $(k, k)$, $(k, k+1)$, and $(2 k+1,2 k)$ are always realizable and that $(2,1)$ is not. We complete the characterization of realizable pairs by showing that no pair of the form $(k, k+2)$ is realizable (confirming Conjecture 1 in [2]) and that $(2 k, 2 k-1$ ) is realizable whenever $k \geq 2$ (refuting Conjecture 2 in [2]).

Non-realizability of $(k, k+2)$ follows easily from the Continuation Principle. The intuition behind the proof below was suggested by Kevin Milans, who proposed that Dominator should simply ignore Staller's first move and proceed as in the Dominator-start game.

Corollary 4.1. For every graph $G$, we have $\gamma_{g}^{\prime}(G) \leq \gamma_{g}(G)+1$.

Proof. Consider the Staller-start game on $G$. Let $v$ be an optimal first move for Staller and let $G^{\prime}$ be the resulting partially-dominated graph. By the Continuation Principle, $\gamma_{g}\left(G^{\prime}\right) \leq$ $\gamma_{g}(G)$, so $\gamma_{g}^{\prime}(G)=\gamma_{g}\left(G^{\prime}\right)+1 \leq \gamma_{g}(G)+1$.

Together with Theorem 6 in [2], this yields the following result.

Corollary 4.2. For every graph $G$, we have $\left|\gamma_{g}^{\prime}(G)-\gamma_{g}(G)\right| \leq 1$.

We next refute Conjecture 2 in [2] by showing that $(2 k, 2 k-1)$ is realizable for $k \geq 2$.

Proposition 4.3. When $k \geq 2$, there is a graph $G_{k}$ with $\gamma_{g}\left(G_{k}\right)=2 k$ and $\gamma_{g}^{\prime}\left(G_{k}\right)=2 k-1$.

Proof. Let $G_{2}$ be $P_{4} \square P_{2}$ (shown on the left in Figure 1). For $k \geq 3$, define $G_{k}$ recursively as the disjoint union of $G_{k-1}$ and $C_{4}$. As argued in [2], we have $\gamma_{g}\left(G_{k}\right)=\gamma_{g}\left(G_{k-1}\right)+2$ and $\gamma_{g}^{\prime}\left(G_{k}\right)=\gamma_{g}^{\prime}\left(G_{k-1}\right)+2$. Hence it suffices to prove $\gamma_{g}\left(G_{2}\right)=4$ and $\gamma_{g}^{\prime}\left(G_{2}\right)=3$.

We first show $\gamma_{g}\left(G_{2}\right)=4$. For the lower bound, we give a strategy for Staller. Consider Dominator's first move. If Dominator plays a vertex of degree 2, then in response Staller plays its neighbor of degree 2 ; the four remaining undominated vertices cannot all be dominated in one move. If instead Dominator plays a vertex of degree 3, then in response Staller plays the vertex of degree 3 not adjacent to it; again the two remaining undominated vertices cannot both be dominated in one move.

For the upper bound, it suffices for Dominator to play, on the first and third turns, a vertex of degree 2 and the vertex of degree 3 at distance 3 from it. This leaves only one vertex undominated, which Staller must dominate on the fourth turn. (Recall from Remark 2.2 that we need not require that Dominator's second move actually dominate new vertices, so Staller's intervening move is irrelevant.) 
We next show $\gamma_{g}^{\prime}\left(G_{2}\right)=3$. For the lower bound, $\gamma_{g}^{\prime}\left(G_{2}\right) \geq \gamma\left(G_{2}\right)=3$. For the upper bound, observe that no matter how Staller plays, Dominator can respond so as to leave only one undominated vertex, which Staller must subsequently dominate on the next turn.

The examples constructed above are, in general, disconnected. A construction by Zamani yields connected graphs $H_{k}$ that also realize these pairs. To form $H_{k}$, start with one copy of $P_{4} \square P_{2}$ and $k-2$ copies of $P_{3} \square P_{2}$. Let $y_{0}$ be a vertex of degree 2 in the copy of $P_{4} \square P_{2}$. In the $i$ th copy of $P_{3} \square P_{2}$, let $x_{i}$ and $y_{i}$ be vertices of degree 2 with a common neighbor. Form $H_{k}$ by identifying $y_{i}$ with $x_{i+1}$ for $i \in\{0, \ldots, k-3\}$. (See Figure 1.) The proof that $H_{k}$ realizes $(2 k, 2 k-1)$ is quite complex; we refer the interested reader to [14]. More recently, Košmrlj [12] provided 2-connected constructions.
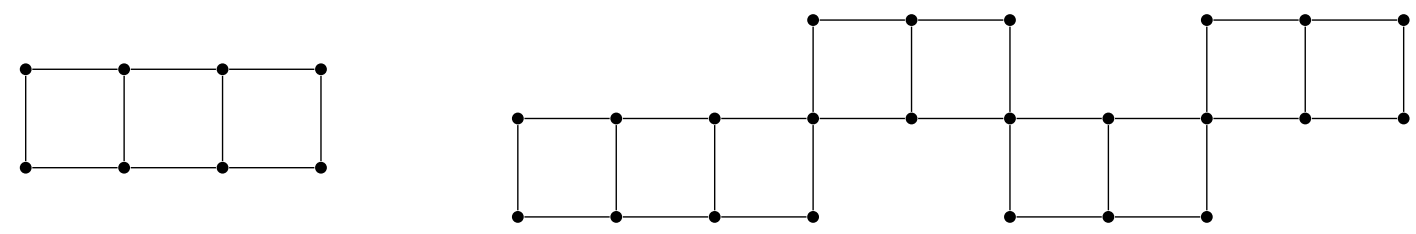

Figure 1. Left: the graph $G_{2}$. Right: the graph $H_{5}$.

In [3], the authors also conjectured that $(k, k-1)$ cannot be realized by a tree.

Conjecture 4.4. [3] There is no tree $T$ such that $\gamma_{g}^{\prime}(T)=\gamma_{g}(T)-1$.

In light of Corollary 4.1, this amounts to proving that $\gamma_{g}(T) \leq \gamma_{g}^{\prime}(T)$ for all trees $T$. We next prove this conjecture. To facilitate induction, we consider all partially-dominated forests, instead of just trees; this yields a stronger statement. We begin with an easy lemma. Note that $G+H$ denotes the disjoint union of graphs $G$ and $H$.

Lemma 4.5. Given $\ell \geq 2$, assume $\gamma_{g}(F) \leq \gamma_{g}^{\prime}(F)$ for all partially-dominated forests $F$ such that $\gamma_{g}(F) \leq \ell$. If $G$ is such a forest, then $\gamma_{g}\left(G+K_{1}\right)>\gamma_{g}(G)$ and $\gamma_{g}^{\prime}\left(G+K_{1}\right)>\gamma_{g}^{\prime}(G)$.

Proof. Fixing $\ell$, we use induction on the number of undominated vertices in $G$. The claim is trivial if $G$ has no undominated vertices.

To prove $\gamma_{g}\left(G+K_{1}\right)>\gamma_{g}(G)$, let $v$ be an optimal first move in the Dominator-start game on $G+K_{1}$. If $v$ is the added vertex, then $\gamma_{g}\left(G+K_{1}\right)=1+\gamma_{g}^{\prime}(G) \geq 1+\gamma_{g}(G)$. Otherwise, $v$ belongs to $G$; let $G_{v}$ denote the partially-dominated graph obtained from $G$ by dominating $N[v]$. By optimality of $v$ we have $\gamma_{g}\left(G+K_{1}\right)=1+\gamma_{g}^{\prime}\left(G_{v}+K_{1}\right)$; by the Continuation Principle, $\gamma_{g}\left(G_{v}\right) \leq \gamma_{g}(G) \leq \ell$. These observations and the induction hypothesis yield

$$
\gamma_{g}\left(G+K_{1}\right)=1+\gamma_{g}^{\prime}\left(G_{v}+K_{1}\right)>1+\gamma_{g}^{\prime}\left(G_{v}\right) .
$$

Since Dominator does at least as well by playing optimally as by playing $v$ first, $1+\gamma_{g}^{\prime}\left(G_{v}\right) \geq$ $\gamma_{g}(G)$ and hence $\gamma_{g}\left(G+K_{1}\right)>\gamma_{g}(G)$. 
To prove $\gamma_{g}^{\prime}\left(G+K_{1}\right) \leq \gamma_{g}^{\prime}(G)$, let $v$ be an optimal first move in the Staller-start game on $G$. Now

$$
\gamma_{g}^{\prime}\left(G+K_{1}\right) \geq 1+\gamma_{g}\left(G_{v}+K_{1}\right)>1+\gamma_{g}\left(G_{v}\right)=\gamma_{g}^{\prime}(G),
$$

with the strict inequality following from the induction hypothesis and the equality following from optimality of $v$.

We now prove the conjecture itself.

Theorem 4.6. If $F$ is a partially-dominated forest, then $\gamma_{g}(F) \leq \gamma_{g}^{\prime}(F)$.

Proof. Since always $\left|\gamma_{g}(F)-\gamma_{g}^{\prime}(F)\right| \leq 1$ (Corollary 4.2), it suffices to show that $\gamma_{g}(F)=k$ and $\gamma_{g}^{\prime}(F)=k-1$ cannot both hold. This is clear when $k \leq 2$; we proceed by induction on $k$. For $k \geq 3$, suppose that $\gamma_{g}(F)=k$ and $\gamma_{g}^{\prime}(F)=k-1$. For $v \in V(F)$, let $F_{v}$ be the partially-dominated forest obtained from $F$ by dominating $N[v]$. Since $\gamma_{g}^{\prime}(F)=k-1$, Dominator can force the game to end within $k-2$ more turns after any first move by Staller; hence $\gamma_{g}\left(F_{v}\right) \leq k-2$. Similarly, $\gamma_{g}(F)=k$ implies $\gamma_{g}^{\prime}\left(F_{v}\right) \geq k-1$. By Corollary 4.2, it now follows that $\gamma_{g}\left(F_{v}\right)=k-2$ and $\gamma_{g}^{\prime}\left(F_{v}\right)=k-1$ for any $v \in V(F)$.

Let $C$ be a component of $F$ having an undominated vertex. Fix a root vertex $r$ in $C$, and let $x$ be an undominated vertex in $C$ farthest from $r$. If $x \neq r$, then let $y$ be the parent of $x$, otherwise $y=x$. Let $u$ be an optimal first move in the Staller-start game on $F_{y}$. By the choice of $x$, all descendants of $x$ are dominated in $F$; by definition, $y$ and its neighbors are all dominated in $F_{y}$. Thus no vertex in $N[x]$ is a legal move, so $u \notin N[x]$ and $x \notin N[u]$.

Denote by $F_{y, u}$ the partially-dominated graph obtained from $F_{y}$ by dominating all of $N[u]$. By the choice of $u$ and the fact that $\gamma_{g}^{\prime}\left(F_{y}\right)=k-1$, we have $\gamma_{g}\left(F_{y, u}\right)=k-2$. However, we have also shown that $\gamma_{g}\left(F_{u}\right)=k-2$. Let $F_{u}^{\prime}$ be the partially-dominated forest obtained from $F_{u}$ by dominating $N[y]-x$; note that $F_{u}^{\prime}$ and $F_{y, u}$ are identical except that $x$ is dominated in the latter but not in the former.

By the choice of $x$, all neighbors of $x$ and vertices at distance 2 from $x$ are dominated. Thus, a move on $F_{u}^{\prime}$ that dominates $x$ cannot dominate any other vertices; it serves only to dominate $x$. Thus $\gamma_{g}\left(F_{u}^{\prime}\right)=\gamma_{g}\left(F_{y, u}+K_{1}\right)$. Now the Continuation Principle and Lemma 4.5 together yield

$$
k-2=\gamma_{g}\left(F_{u}\right) \geq \gamma_{g}\left(F_{u}^{\prime}\right)=\gamma_{g}\left(F_{y, u}+K_{1}\right)>\gamma_{g}\left(F_{y, u}\right)=k-2,
$$

contradicting the choice of $F$.

When Staller has the option to pass, doing so has the effect of choosing to play second on the current partially-dominated graph. Theorem 4.6 shows that Staller never benefits from playing second on a partially-dominated forest. This observation yields a useful corollary:

Corollary 4.7. If $F$ is a partially-dominated forest, then $\hat{\gamma}_{g}(F)=\gamma_{g}(F)$ and $\hat{\gamma}_{g}^{\prime}(F)=\gamma_{g}^{\prime}(F)$. 
We close this section with an additional example related to realizability questions. We have noted the trivial upper bound $\gamma_{g}(G) \leq 2 \gamma(G)-1$ from [2]. Equality holds for complete graphs, where both sides equal 1 . The sharpness examples in [2] are more general, obtained by attaching many pendant edges to each vertex of a complete graph. We note that attaching about $\log _{2} r$ pendant edges to each vertex of $K_{r}$ yields sharpness in the bound for an $n$-vertex graph whose domination number is about $n / \log _{2} n$. Thus one can achieve equality with high connectivity and domination number 1 or with high domination number and connectivity 1. Letting $n$ denote the number of vertices, we construct such examples with domination number and connectivity both approximately $\sqrt{n}$.

Proposition 4.8. For $k \in \mathbb{N}$, there is a $k$-connected graph $G$ with $k(k+1)$ vertices such that $\gamma_{g}(G)=2 \gamma(G)-1$.

Proof. Let $G=K_{1, k} \square K_{k}$. Viewing the the $K_{1, k}$-fibers as "rows" and the $K_{k}$-fibers as "columns", we may write $V(G)=\left\{w_{i, j}: 1 \leq i \leq k, 1 \leq j \leq k+1\right\}$, where $w_{i, j}$ denotes the vertex in row $i$, column $j$. For convenience, let $u_{i}=w_{i, 1}$ for $1 \leq i \leq k$, let $U=\left\{u_{1}, \ldots, u_{k}\right\}$, and let $W=V(G)-U$. See Figure 2 for the case $k=4$.

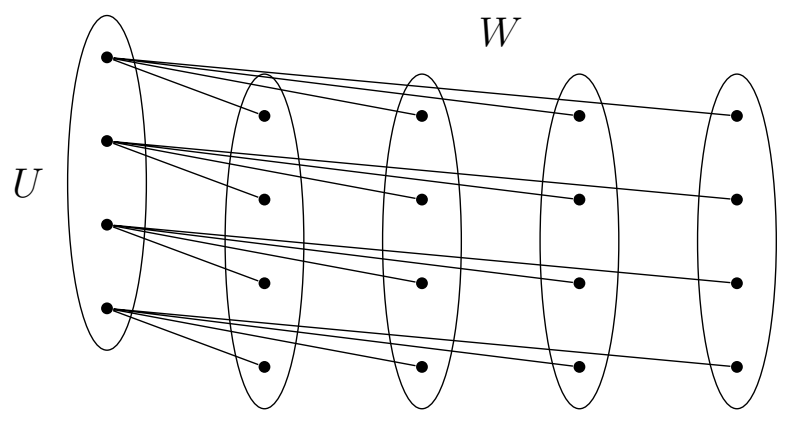

Figure 2. The graph $G$ (for $k=4$ ).

The $k$ vertices in $U$ form a dominating set for $G$. Any set $S$ having at most $k-1$ vertices omits some $u_{i} \in U$ and the entire $j$ th column in $W$ for some $j$. The set $S$ does not dominate $w_{i, j}$; hence $\gamma(G)=k$.

Each of the $k$ rows is a connected dominating set. A separating set must contain at least one vertex from each connected dominating set, so $G$ is $k$-connected. Note that $U$ is a separating $k$-set.

Finally, we present a Staller strategy to prove that $\gamma_{g}(G) \geq 2 k-1$. On each round, if Dominator does not play in $U$, then Staller plays some vertex in $U$. If Dominator plays $u_{i}$, then Staller finds a column $j$ in which no vertex has been played and plays $w_{i, j}$.

Consider an instance of the game with Staller playing this strategy. Let $d_{i}$ be the $i$ th vertex that Dominator plays, and let $s_{i}$ be the $i$ th vertex that Staller plays. Under the specified strategy, each pair $\left(d_{i}, s_{i}\right)$ adds 1 to the number of played vertices in $U$, and it 
decreases by at most 1 the number of columns in $W$ containing no played vertices. Thus, after Staller has played $s_{i}$, there are $k-i$ unplayed vertices in $U$ and at least $k-i$ columns in $W$ with no played vertices. If $i<k$, then there is an unplayed vertex $u \in U$ and a column of $W$ in which no vertex has been played. The neighbor of $u$ in this column is undominated, so the game continues. Dominator therefore will have to play a $k$ th time, at which point $2 k-1$ moves have been played.

\section{Forests}

In this section, we study the maximum values of $\gamma_{g}$ and $\gamma_{g}^{\prime}$ over $n$-vertex isolate-free forests. Although we have not determined the maxima exactly, we make the following conjecture:

Conjecture 5.1. If $G$ is an isolate-free $n$-vertex forest, then $\gamma_{g}(G) \leq \frac{3 n}{5}$ and $\gamma_{g}^{\prime}(G) \leq \frac{3 n+2}{5}$.

We first show that these bounds would be sharp. Recall that in a partially-dominated graph, a saturated vertex is a vertex $v$ such that all of $N[v]$ is dominated, and the residual graph is formed by discarding all saturated vertices and all edges joining dominated vertices.

Proposition 5.2. Let $k$ be a positive integer. If $G$ is obtained from a $k$-vertex graph by attaching two pendant paths of length 2 to each vertex, then $\gamma_{g}(G)=3 k$. If $G^{\prime}$ is obtained from a $(k+1)$-vertex graph by attaching two pendant paths of length 2 to each of $k$ vertices, then $\gamma_{g}^{\prime}\left(G^{\prime}\right)=3 k+1$.

Proof. We may view $G$ as consisting of $k$ copies of $P_{5}$ plus some edges joining the centers. Let $H$ be the partially-dominated graph corresponding to $G$ with the centers of the copies of $P_{5}$ dominated, and let $H^{\prime}$ be its residual graph; note that $H^{\prime}$ is just $k P_{5}$ with the centers of the copies of $P_{5}$ dominated. Now $\gamma_{g}(G) \geq \gamma_{g}\left(H^{\prime}\right)$ by the Continuation Principle, so to show $\gamma_{g}(G) \geq 3 k$ it suffices to show $\gamma_{g}\left(H^{\prime}\right) \geq 3 k$.

We present a Staller strategy that enforces this lower bound. On each turn, Staller responds to Dominator's previous move. Let $v$ be that move, and let $w$ be the center of the copy of $P_{5}$ containing $v$. If $w$ is a legal move, then Staller plays it. Otherwise, Staller plays the center of some other $P_{5}$ if possible, and plays any legal move if not. Staller's strategy ensures that the first or the second vertex played in each copy of $P_{5}$ is the center. Hence at least three vertices must be played in each copy of $P_{5}$, so $\gamma_{g}\left(H^{\prime}\right) \geq 3 k$.

It now follows easily that $\gamma_{g}^{\prime}\left(G^{\prime}\right) \geq 3 k+1$. On the first turn, Staller plays the lone original vertex not extended to a copy of $P_{5}$. Operating on the resulting residual graph and applying the Continuation Principle now yields $\gamma_{g}^{\prime}\left(G^{\prime}\right) \geq 1+\gamma_{g}\left(H^{\prime}\right) \geq 3 k+1$.

Dominator can enforce matching upper bounds with the same strategy: if Staller plays vertex $v$, then Dominator plays the center of the copy of $P_{5}$ containing $v$, the center of some other copy of $P_{5}$, or any legal move, in order of preference. This ensures that no more than 
three vertices in any one copy of $P_{5}$ are played. Thus $\gamma_{g}(G) \leq 3 k$ and $\gamma_{g}^{\prime}\left(G^{\prime}\right) \leq 3 k+1$ (since on $G^{\prime}$ the vertex not extended to a copy of $P_{5}$ might also be played).

The graphs $G$ and $G^{\prime}$ in the hypotheses of Proposition 5.2 can be taken to be trees, so for each $k$ there exists a $5 k$-vertex tree $T$ with $\gamma_{g}(T)=3 k=\frac{3(5 k)}{5}$. Similarly, for each $k$ there exists a $(5 k+1)$-vertex tree $T^{\prime}$ with $\gamma_{g}^{\prime}(T)=3 k+1=\frac{3(5 k+1)+2}{5}$. Thus the bounds in Conjecture 5.1 cannot be strengthened.

We devote the remainder of this section to partial progress toward Conjecture 5.1. First we prove an easy upper bound on $\gamma_{g}$ and $\gamma_{g}^{\prime}$ that holds for the more general family of isolatefree chordal graphs (chordal graphs are those having no cycle of length at least 4 as an induced subgraph). The proof idea is to bound the length of the game by ensuring that vertices become saturated "quickly enough".

A simplicial vertex in a graph is any vertex whose neighborhood is a clique; it is wellknown that every chordal graph has a simplicial vertex.

Proposition 5.3. If $G$ is a partially-dominated isolate-free n-vertex chordal graph, then $\gamma_{g}(G) \leq \frac{2 n}{3}$ and $\gamma_{g}^{\prime}(G) \leq \frac{2 n}{3}$.

Proof. We proceed by induction on $n$. The claim is trivial when $n \leq 2$, so assume $n \geq 3$.

For the bound on $\gamma_{g}(G)$, let $v$ be a simplicial vertex in $G$. Since $G$ has no isolated vertices, $v$ has a neighbor, $w$. Dominator plays $w$; since $v$ is simplicial, $N[v] \subseteq N[w]$, so this move saturates both $v$ and $w$. If this move finishes the game, then $\gamma_{g}(G)=1$.

Otherwise, Staller's subsequent move saturates at least one more vertex. Let $G^{\prime}$ be the graph obtained from $G$ by removing all saturated vertices. (Since removing edges from a chordal graph can destroy chordality, we retain edges joining dominated vertices that are not saturated.) These moves saturated at least three vertices, so $G^{\prime}$ has at most $n-3$ vertices. Moreover, since every induced subgraph of a chordal graph is chordal, $G^{\prime}$ is chordal. For a vertex $v$ to remain in $G^{\prime}$, either $v$ or one of its neighbors must be undominated and hence unsaturated, so $G^{\prime}$ is isolate-free. Thus the induction hypothesis applies to $G^{\prime}$, and

$$
\gamma_{g}(G) \leq 2+\gamma_{g}\left(G^{\prime}\right) \leq 2+\frac{2(n-3)}{3}=\frac{2 n}{3} .
$$

The bound on $\gamma_{g}^{\prime}(G)$ follows similarly.

When we apply the induction hypothesis to a smaller partially-dominated residual graph to obtain upper bounds, the Continuation Principle (Lemma 2.1) allows us to ignore which vertices in the residual graph are already dominated. We use this observation freely.

We next show that the upper bound in Conjecture 5.1 holds for those isolate-free forests in which each component is a caterpillar. Here the Segmentation Lemma yields a straightforward inductive proof. To apply the Segmentation Lemma directly, we would need to prove upper bounds on $\hat{\gamma}_{g}$ and $\hat{\gamma}_{g}^{\prime}$, as in Theorem 3.4. However, the following lemma, an immediate 
consequence of the Segmentation Lemma and Corollary 4.7, allows us to work with $\gamma_{g}$ and $\gamma_{g}^{\prime}$ directly, which slightly simplifies the arguments.

Lemma 5.4. Let $F, F_{1}$, and $F_{2}$ be partially-dominated forests. If $F=F_{1} \cup F_{2}$, then $\gamma_{g}(F) \leq$ $\gamma_{g}\left(F_{1}\right)+\gamma_{g}^{\prime}\left(F_{2}\right)$ and $\gamma_{g}^{\prime}(F) \leq \gamma_{g}^{\prime}\left(F_{1}\right)+\gamma_{g}^{\prime}\left(F_{2}\right)$.

Theorem 5.5. If $F$ is an isolate-free $n$-vertex forest in which each component is a caterpillar, then $\gamma_{g}(F) \leq \frac{3 n}{5}$ and $\gamma_{g}^{\prime}(F) \leq \frac{3 n+2}{5}$.

Proof. We prove both bounds simultaneously by induction on $n$. To facilitate induction, we generalize the claim by allowing $F$ to be partially-dominated. When $n \leq 5$ the claim is clear, so assume $n \geq 6$.

Suppose that Staller plays first, and let $v$ be an optimal first move. This move saturates $v$, so the resulting residual graph $F^{\prime}$ has at most $n-1$ vertices. Thus

$$
\gamma_{g}^{\prime}(F)=1+\gamma_{g}\left(F^{\prime}\right) \leq 1+\frac{3(n-1)}{5}=\frac{3 n+2}{5} .
$$

Now suppose that Dominator plays first. If $F$ has a vertex adjacent to two leaves, then Dominator plays it; the resulting residual graph $F^{\prime}$ has at most $n-3$ vertices, so

$$
\gamma_{g}(F) \leq 1+\gamma_{g}^{\prime}\left(F^{\prime}\right) \leq 1+\frac{3(n-3)+2}{5}=\frac{3 n-2}{5}<\frac{3 n}{5} .
$$

If $F$ has a component $C$ isomorphic to $P_{2}$, then Lemma 5.4 yields

$$
\gamma_{g}(F) \leq \gamma_{g}(F-V(C))+\gamma_{g}^{\prime}\left(P_{2}\right) \leq \frac{3(n-2)}{5}+1=\frac{3 n-1}{5}<\frac{3 n}{5}
$$

Similarly, if $F$ has a component $C$ isomorphic to $P_{4}$ or $P_{5}$, then Lemma 5.4 yields $\gamma_{g}(F) \leq$ $(3 n-2) / 5$ or $\gamma_{g}(F) \leq 3 n / 5$, respectively, since $\gamma_{g}^{\prime}\left(P_{4}\right)=2$ and $\gamma_{g}^{\prime}\left(P_{5}\right)=3$.

Now suppose that $F$ has no vertex adjacent to two leaves and no component isomorphic to $P_{2}, P_{4}$, or $P_{5}$; these assumptions together imply that no component of $F$ has fewer than six vertices. Let $C$ be a component of $F$, and let $v_{1}, \ldots, v_{k}$ be the vertices of some longest path in $C$, in order. Let $i$ be the least index such that $d\left(v_{i}\right)=3$, if such an index exists; otherwise, let $i=6$. If $i \leq 5$, then let $x$ be the leaf adjacent to $v_{i}$. Since $v_{1}$ is a leaf, $i \neq 1$; since $C$ has no vertex adjacent to two leaves, $i \neq 2$. If $i=3$, then Dominator plays $v_{3}$. The residual graph has the form $P_{2}+F^{\prime}$, where $F^{\prime}$ has $n-4$ vertices (since it omits $v_{1}, v_{2}, v_{3}$, and $x$ ). Now Lemma 5.4 yields

$$
\gamma_{g}(F) \leq 1+\gamma_{g}^{\prime}\left(P_{2}+F^{\prime}\right) \leq 1+\gamma_{g}^{\prime}\left(P_{2}\right)+\gamma_{g}^{\prime}\left(F^{\prime}\right) \leq 1+1+\frac{3(n-4)+2}{5}=\frac{3 n}{5} .
$$

If $i=4$ or $i=5$, then let $S=\left\{v_{1}, v_{2}, v_{3}, v_{4}, x\right\}$, and let $F^{\prime}=F-S$. Now Lemma 5.4 yields

$$
\gamma_{g}(F) \leq \gamma_{g}\left(F^{\prime}\right)+\gamma_{g}^{\prime}\left(F\left[S \cup\left\{v_{5}\right\}\right]\right) \leq \frac{3(n-5)}{5}+3=\frac{3 n}{5}
$$


Finally, if $i>5$ or if $C$ has no vertex with degree at least 3 , then let $S=\left\{v_{1}, v_{2}, v_{3}, v_{4}, v_{5}\right\}$ and let $F^{\prime}=F-S$. Now Lemma 5.4 yields

$$
\gamma_{g}(F) \leq \gamma_{g}\left(F^{\prime}\right)+\gamma_{g}^{\prime}\left(F\left[S \cup\left\{v_{6}\right\}\right]\right) \leq \frac{3(n-5)}{5}+3=\frac{3 n}{5} .
$$

Using this approach and much more intricate case analysis, we next obtain weaker bounds on $\gamma_{g}$ and $\gamma_{g}^{\prime}$ over the class of all isolate-free forests.

Theorem 5.6. If $F$ is an isolate-free $n$-vertex forest, then $\gamma_{g}(F) \leq \frac{7 n}{11}$ and $\gamma_{g}^{\prime}(F) \leq \frac{7 n+4}{11}$.

Proof. We prove both bounds simultaneously by induction on $n$. To facilitate induction, we generalize the claim by allowing $F$ to be partially-dominated. Both bounds hold trivially when $F$ has no vertices, so assume $n \geq 1$.

Suppose Staller plays first, and let $F^{\prime}$ be the resulting residual graph; by the induction hypothesis,

$$
\gamma_{g}^{\prime}(F)=1+\gamma_{g}\left(F^{\prime}\right) \leq 1+\frac{7(n-1)}{11}=\frac{7 n+4}{11}
$$

We may therefore assume that Dominator moves first.

Using the induction hypothesis, we may argue as in the proof of Theorem 5.5 that $F$ has no vertex adjacent to two leaves and no component isomorphic to $P_{2}, P_{4}$, or $P_{5}$.

For $v \in V(F)$, define a tail of $v$ to be a nontrivial path whose endpoints are $v$ and some leaf of $F$, and whose internal vertices all have degree 2 in $F$. We make several reductions based on the lengths of tails in $F$. We exclude each case by obtaining an upper bound for $\gamma_{g}(F)$ that is strictly less than $7 n / 11$.

First suppose that some vertex $v$ has a tail with length at least 5 . Let the vertices of this tail be $w_{0}, \ldots, w_{l}$, in order, with $w_{l}=v$. Let $F_{1}$ be the copy of $P_{6}$ induced by $\left\{w_{0}, \ldots, w_{5}\right\}$ and let $F_{2}=F-\left\{w_{0}, \ldots, w_{4}\right\}$. By Lemma 5.4,

$$
\gamma_{g}(F) \leq \gamma_{g}\left(F_{2}\right)+\gamma_{g}^{\prime}\left(F_{1}\right) \leq \frac{7(n-5)}{11}+3=\frac{7 n-2}{11}
$$

Thus we may assume that every tail in $F$ has length at most 4.

Next suppose that some vertex $v$ has at least two tails. Let $W$ and $X$ be two tails of $v$, with $l_{w}$ and $l_{x}$ their respective lengths and $l_{w} \geq l_{x}$. Since $l_{w} \leq 4$, we have $l_{w}+l_{x} \leq 8$. Let $F_{1}=W \cup X$ and let $F_{2}=F-\left(F_{1}-v\right)$; note that $F_{1} \cong P_{l_{w}+l_{x}+1}$. We now apply Lemma 5.4, along with the observations that $\gamma_{g}^{\prime}\left(P_{6}\right)=3, \gamma_{g}\left(P_{7}\right)=3, \gamma_{g}^{\prime}\left(P_{8}\right)=4$, and $\gamma_{g}^{\prime}\left(P_{9}\right)=5$. If $l_{w}+l_{x} \in\{5,7,8\}$, then $\gamma_{g}(F) \leq \gamma_{g}\left(F_{2}\right)+\gamma_{g}^{\prime}\left(F_{1}\right) \leq 7 n / 11$ in all cases. If instead $l_{w}+l_{x}=6$, then $\gamma_{g}(F) \leq \gamma_{g}\left(F_{1}\right)+\gamma_{g}^{\prime}\left(F_{2}\right) \leq 7 n / 11$. Thus $l_{w}+l_{x} \leq 4$.

If $l_{w}=l_{x}=1$, then $v$ is adjacent to two leaves, which is already excluded. If $l_{w}=2$ and $l_{x}=1$, then Dominator plays $v$; the residual graph has the form $P_{2}+F_{1}$ for some forest $F_{1}$ with at most $n-4$ vertices. Lemma 5.4 now yields

$$
\gamma_{g}(F) \leq 1+\gamma_{g}^{\prime}\left(P_{2}+F_{1}\right) \leq 1+\gamma_{g}^{\prime}\left(P_{2}\right)+\gamma_{g}^{\prime}\left(F_{1}\right) \leq 1+1+\frac{7(n-4)+4}{11}=\frac{7 n-2}{11} .
$$


If $l_{w}=3$ and $l_{x}=1$, then Dominator again plays $v$. The residual graph now has the form $P_{3}+F_{1}$. If Staller next plays on $F_{1}$, then Dominator responds by completely dominating the copy of $P_{3}$. The resulting residual graph $F_{1}^{\prime}$ has at most $n-6$ vertices, so

$$
\gamma_{g}(F) \leq 3+\gamma_{g}^{\prime}\left(F_{1}^{\prime}\right) \leq 3+\frac{7(n-6)+4}{11}=\frac{7 n-5}{11}
$$

Alternatively, if Staller next plays on the copy of $P_{3}$, then the resulting residual graph has the form $F_{1}+F_{2}$, where $F_{2}$ is the null graph or $P_{2}$. Lemma 5.4 now yields

$$
\gamma_{g}(F) \leq 2+\gamma_{g}\left(F_{1}+F_{2}\right) \leq 2+\gamma_{g}\left(F_{1}\right)+\gamma_{g}^{\prime}\left(F_{2}\right) \leq 2+\frac{7(n-5)}{11}+1=\frac{7 n-2}{11}
$$

The only remaining case is $l_{w}=l_{x}=2$, so we may assume that every tail of $v$ has length 2. Suppose that $v$ has a third tail, $Y$. Dominator plays $v$; the resulting residual graph has the form $F_{1}+3 P_{2}$. Lemma 5.4 now yields

$$
\gamma_{g}(F) \leq 1+\gamma_{g}^{\prime}\left(F_{1}+3 P_{2}\right) \leq 1+\gamma_{g}^{\prime}\left(F_{1}\right)+\gamma_{g}^{\prime}\left(3 P_{2}\right) \leq 1+\frac{7(n-7)+4}{11}+3=\frac{7 n-1}{11} .
$$

By these reductions, we may assume that for each $v \in V(F)$, one of the following holds:

- $v$ has no tails.

- $v$ has exactly one tail, which has length at most 4 .

- $v$ has exactly two tails, both with length 2 .

We consider the remaining cases with this restriction in place.

Let $C$ be a component of $F$. We have argued that $C \notin\left\{P_{2}, P_{3}, P_{4}, P_{5}\right\}$; since no vertex in $C$ has a tail with length at least 5 , also $C$ is not $P_{k}$ for $k \geq 6$. Thus $C$ has a branch vertex (that is, a vertex with degree at least 3). Let $r$ be a leaf of $C$, and view $C$ as a rooted tree with root $r$. Let $v$ be a branch vertex of $C$ farthest from $r$. Since no descendant of $v$ is a branch vertex, it follows that $v$ has exactly two tails, both with length 2 . Let $u$ be the parent of $v$. (Note that $v \neq r$, since $r$ is a leaf and $v$ is not; hence $v$ has a parent.)

If $u=r$, then $C$ is the graph formed from $P_{5}$ by attaching a pendant leaf to the center vertex. Thus $\gamma_{g}(C)=3$, so by Lemma 5.4 ,

$$
\gamma_{g}(F) \leq \gamma_{g}(C)+\gamma_{g}^{\prime}(F-V(C)) \leq 3+\frac{7(n-6)+4}{11}=\frac{7 n-5}{11}
$$

We may therefore assume $u \neq r$. We break the remaining argument into eight cases.

Case 1: Some child $w$ of $u$ is a branch vertex other than $v$. It follows from the choice of $v$ that $w$ has no descendant branch vertices. Thus $w$ has two descendant tails of length 2 


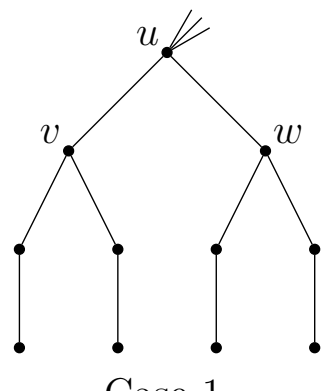

Case 1

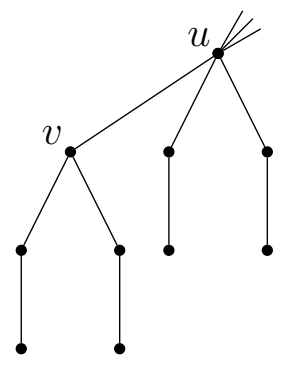

Case 2

\section{Figure 3}

and no other descendants. Let $F_{1}$ consist of $u$ and everything below as drawn in Figure 3 ; let $F_{2}=F-\left(F_{1}-u\right)$. Now Lemma 5.4 and the observation that $\gamma_{g}\left(F_{1}\right)=6$ yield

$$
\gamma_{g}(F) \leq \gamma_{g}\left(F_{1}\right)+\gamma_{g}^{\prime}\left(F_{2}\right) \leq 6+\frac{7(n-10)+4}{11}=\frac{7 n}{11}
$$

Case 2: $u$ has two tails. Dominator plays $u$. The resulting residual graph has the form $F_{1}+2 P_{2}+P_{5}$, where $F_{1}$ has at most $n-10$ vertices. Now Lemma 5.4 yields

$\gamma_{g}(F) \leq 1+\gamma_{g}^{\prime}\left(F_{1}+2 P_{2}+P_{5}\right) \leq 1+\gamma_{g}^{\prime}\left(F_{1}\right)+\gamma_{g}^{\prime}\left(2 P_{2}+P_{5}\right) \leq 1+\frac{7(n-10)+4}{11}+5=\frac{7 n}{11}$

Case 3: u has one descendant tail, $X$.

Case 3a: $X$ has length 1. Dominator plays $u$. The resulting residual graph has the form $F_{1}+P_{5}$, where $F_{1}$ has at most $n-7$ vertices. Now

$$
\gamma_{g}(F) \leq 1+\gamma_{g}^{\prime}\left(F_{1}+P_{5}\right) \leq 1+\gamma_{g}^{\prime}\left(F_{1}\right)+\gamma_{g}^{\prime}\left(P_{5}\right) \leq 1+\frac{7(n-7)+4}{11}+3=\frac{7 n-1}{11}
$$

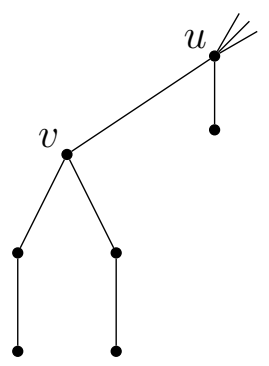

Case 3a

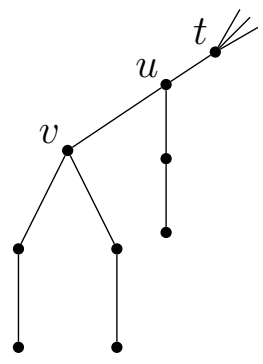

Case 3b

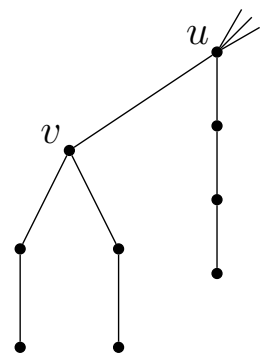

Case 3c

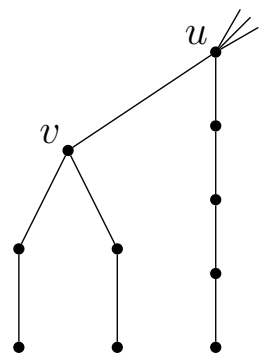

Case 3d

Figure 4 
Case 3b: $X$ has length 2. Let $t$ be the parent of $u$. Let $F_{1}$ consist of $t$ and everything below as drawn in Figure 4 ; let $F_{2}=F-\left(F_{1}-t\right)$. Now $\gamma_{g}^{\prime}\left(F_{1}\right)=5$, so

$$
\gamma_{g}(F) \leq \gamma_{g}\left(F_{2}\right)+\gamma_{g}^{\prime}\left(F_{1}\right) \leq \frac{7(n-8)}{11}+5=\frac{7 n-1}{11} .
$$

Case 3c: $X$ has length 3. Let $F_{1}$ consist of $u$ and everything below, as drawn in Figure 4; let $F_{2}=F-\left(F_{1}-u\right)$. Now $\gamma_{g}^{\prime}\left(F_{1}\right)=5$, so the computation is the same as in Case $3 \mathrm{~b}$.

Case 3d: $X$ has length 4. Dominator plays $u$. The resulting residual graph has the form $F_{1}+P_{5}+P_{4}$, where $F_{1}$ has at most $n-10$ vertices. Now

$\gamma_{g}(F) \leq 1+\gamma_{g}^{\prime}\left(F_{1}+P_{5}+P_{4}\right) \leq 1+\gamma_{g}^{\prime}\left(F_{1}\right)+\gamma_{g}^{\prime}\left(P_{5}\right)+\gamma_{g}^{\prime}\left(P_{4}\right) \leq 1+\frac{7(n-10)+4}{11}+3+2=\frac{7 n}{11}$.

We have now considered all cases where $u$ has a child other than $v$. Henceforth, let $t$ be the parent of $u$. We have several more cases to consider.

Case 4: $t=r$. Now $\gamma_{g}(C)=4$, so

$$
\gamma_{g}(F) \leq \gamma_{g}(C)+\gamma_{g}^{\prime}(F-V(C)) \leq 4+\frac{7(n-7)+4}{11}=\frac{7 n-1}{11}
$$

Case 5: $t$ has a child branch vertex, $w$. If $w$ also has a child branch vertex, $x$, then the preceding set of arguments apply with $x$ in place of $v$ to show that $w$ has degree 2 , a contradiction. It follows from this observation and the choice of $v$ that $w$ has no descendant branch vertices. Thus $w$ has two tails, so $C$ is as drawn in Figure 5. Let $F_{1}$ consist of $t$ and everything below in the figure, and let $F_{2}=F-\left(F_{1}-t\right)$. Since $\gamma_{g}^{\prime}\left(F_{1}\right)=7$,

$$
\gamma_{g}(F) \leq \gamma_{g}\left(F_{2}\right)+\gamma_{g}^{\prime}\left(F_{1}\right) \leq \frac{7(n-11)}{11}+7=\frac{7 n}{11}
$$

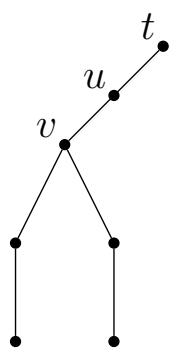

Case 4

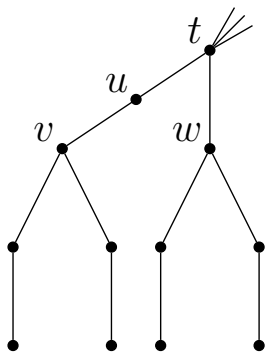

Case 5

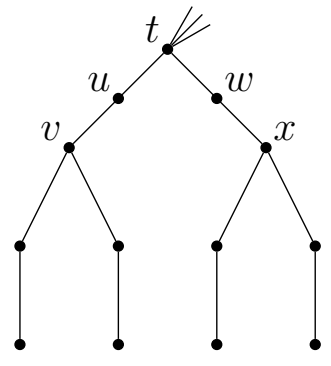

Case 6

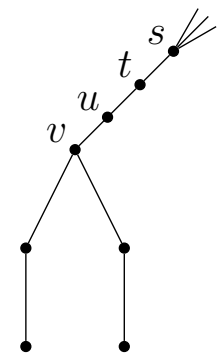

Case 7

Figure 5

Case 6: $t$ has a descendant branch vertex, $x$, other than $v$. Since Case 5 does not apply, $x$ is not a child of $t$. By the choice of $v$, it now follows that $x$ is a grandchild of $t$ and that $x$ has no descendant branch vertices, so it must have two descendant tails. Let $w$ be the 
parent of $x$; now $C$ is as drawn in Case 6 of Figure 5. Let $F_{1}$ consist of $t$ and everything below in the figure, and let $F_{2}=F-\left(F_{1}-t\right)$. Now $\gamma_{g}\left(F_{1}\right)=7$, so

$$
\gamma_{g}(F) \leq \gamma_{g}\left(F_{1}\right)+\gamma_{g}^{\prime}\left(F_{2}\right) \leq 7+\frac{7(n-12)+4}{11}=\frac{7 n-3}{11} .
$$

Case 7: $t$ has degree 2. Let $s$ be the parent of $t$; Dominator plays $s$. The resulting residual graph has the form $F_{1}+F_{2}$, where $F_{1}$ has seven vertices and $F_{2}$ has at most $n-8$ vertices (see Figure 5). If Staller responds on $F_{2}$, then Dominator plays $v$; the residual graph has the form $2 P_{2}+F_{2}^{\prime}$, where $F_{2}^{\prime}$ has at most $n-9$ vertices. Now

$$
\gamma_{g}(F) \leq 3+\gamma_{g}^{\prime}\left(2 P_{2}+F_{2}^{\prime}\right) \leq 3+\gamma_{g}^{\prime}\left(2 P_{2}\right)+\gamma_{g}^{\prime}\left(F_{2}^{\prime}\right) \leq 3+2+\frac{7(n-9)+4}{11}=\frac{7 n-4}{11} .
$$

Suppose instead that Staller responds on $F_{1}$. If Staller plays any vertex other than $t$, then the residual graph has the form $F_{1}^{\prime}+F_{2}$ for some partially-dominated graph $F_{1}^{\prime}$ such that $\gamma_{g}^{\prime}\left(F_{1}^{\prime}\right) \leq 3$. Thus

$$
\gamma_{g}(F) \leq 2+\gamma_{g}\left(F_{1}^{\prime}+F_{2}\right) \leq 2+\gamma_{g}\left(F_{2}\right)+\gamma_{g}^{\prime}\left(F_{1}^{\prime}\right) \leq 2+\frac{7(n-8)}{11}+3=\frac{7 n-1}{11} .
$$

If Staller plays $t$, then Dominator plays a child of $v$. If Staller responds on $F_{1}$, then at most one undominated vertex remains in $F_{1}$, so

$$
\gamma_{g}(F) \leq 4+1+\gamma_{g}\left(F_{2}\right) \leq 5+\frac{7(n-8)}{11}=\frac{7 n-1}{11}
$$

Finally, if Staller plays on $F_{2}$, then Dominator dominates the remainder of $F_{1}$. Now five vertices have been played and the residual graph has at most $n-9$ vertices, so

$$
\gamma_{g}(F) \leq 5+\frac{7(n-9)+4}{11}=\frac{7 n-4}{11}
$$

Case 8: $t$ has a tail, $X$.

Case 8a: $X$ has length 1. Dominator plays $t$. The resulting residual graph has the form $F_{1}+F_{2}$, where $F_{1}$ is the graph formed from $P_{5}$ by attaching a pendant leaf to the center vertex, and $F_{2}$ has at most $n-8$ vertices. If Staller responds on $F_{1}$, then the residual graph has the form $F_{1}^{\prime}+F_{2}$, for some $F_{1}^{\prime}$ such that $\gamma_{g}^{\prime}\left(F^{\prime}\right) \leq 3$. Now

$$
\gamma_{g}(F) \leq 2+\gamma_{g}\left(F_{1}^{\prime}+F_{2}\right) \leq 2+\gamma_{g}^{\prime}\left(F_{1}^{\prime}\right)+\gamma_{g}\left(F_{2}\right) \leq 2+3+\frac{7(n-8)}{11}=\frac{7 n-1}{11}
$$

If instead Staller responds on $F_{2}$, then the residual graph has the form $F_{1}+F_{2}^{\prime}$, for some $F_{2}^{\prime}$ with at most $n-9$ vertices. Now

$$
\gamma_{g}(F) \leq 2+\gamma_{g}\left(F_{1}+F_{2}^{\prime}\right) \leq 2+\gamma_{g}\left(F_{1}\right)+\gamma_{g}^{\prime}\left(F_{2}^{\prime}\right) \leq 2+3+\frac{7(n-9)}{11}=\frac{7 n-8}{11}
$$




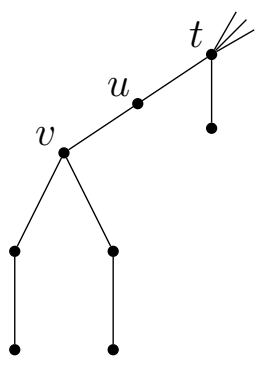

Case $8 \mathrm{a}$

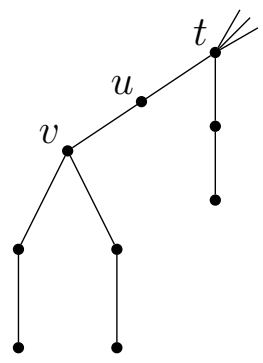

Case $8 b$

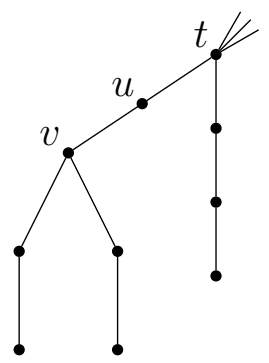

Case 8c

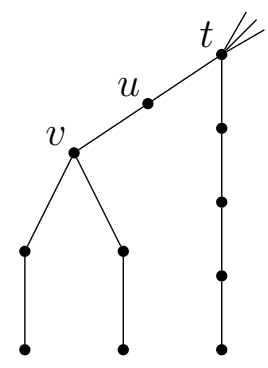

Case $8 \mathrm{~d}$

Figure 6

Case 8b: $X$ has length 2. Let $F_{1}$ consist of $t$ and everything below, as drawn in Figure 6; let $F_{2}=F-\left(F_{1}-t\right)$. Now $\gamma_{g}^{\prime}\left(F_{1}\right)=5$, so

$$
\gamma_{g}(F) \leq \gamma_{g}\left(F_{2}\right)+\gamma_{g}^{\prime}\left(F_{1}\right) \leq \frac{7(n-8)}{11}+5=\frac{7 n-1}{11} .
$$

Case 8c: $X$ has length 3. Again let $F_{1}$ consist of $t$ and everything below, as drawn in Figure 6 . Let $F_{2}=F-\left(F_{1}-t\right)$. Now $\gamma_{g}\left(F_{1}\right)=5$, so

$$
\gamma_{g}(F) \leq \gamma_{g}\left(F_{1}\right)+\gamma_{g}^{\prime}\left(F_{2}\right) \leq 5+\frac{7(n-9)+4}{11}=\frac{7 n-4}{11} .
$$

Case 8d: $X$ has length 4. As before, let $F_{1}$ consist of $t$ and everything below as drawn in Figure 6 ; let $F_{2}=F-\left(F_{1}-t\right)$. Now $\gamma_{g}\left(F_{1}\right)=6$, so

$$
\gamma_{g}(F) \leq \gamma_{g}\left(F_{1}\right)+\gamma_{g}^{\prime}\left(F_{2}\right) \leq 6+\frac{7(n-10)+4}{11}=\frac{7 n}{11} .
$$

We believe that the bound in Theorem 5.6 can be strengthened through more detailed case analysis to yield $\gamma_{g}(F) \leq 5 n / 8$. However, it seems likely that proving Conjecture 5.1 would require stronger techniques.

\section{Open Problems}

Several fundamental questions about the domination game remain unanswered.

While Theorem 5.5 shows that Conjecture 5.1 holds for isolate-free forests in which each component is a caterpillar, the general conjecture remains open.

One could also take a different approach. Theorem 5.5 is best possible, in that equality holds for $k P_{5}$. However, one may ask whether equality holds for arbitrarily large caterpillars. Perhaps restricting to connected tress would permit a tighter bound. 
Question 6.1. Can the upper bound in Theorem 5.5 be tightened on the class of caterpillars?

A comb is a caterpillar formed by attaching a pendant leaf to each vertex of a path. The game domination number of an $n$-vertex comb is asymptotic to $11 n / 20$, so the bound for caterpillars cannot not be made any smaller than this.

The following conjecture would strengthen Theorem 3.4:

Conjecture 6.2. If $G$ is an isolate-free n-vertex graph, then $\gamma_{g}(G) \leq \frac{3 n}{5}$ and $\gamma_{g}^{\prime}(G) \leq \frac{3 n+2}{5}$.

Clearly Conjecture 6.2 implies Conjecture 5.1. One may intially suspect that they are equivalent, but this is not obvious. Unlike many domination parameters, $\gamma_{g}$ is not monotone. There exist graphs $G$ with edges $e$ such that $\gamma_{g}(G-e)<\gamma_{g}(G)$; in fact, $\gamma_{g}(G)-\gamma_{g}(G-e)$ can be as large as 2 . This happens because removing edges from $G$ can eliminate potential moves for Staller, so on the smaller graph Dominator may be able to force Staller to make unfavorable moves. Thus to prove Conjecture 6.2 it would not suffice to apply Conjecture 5.1 to a spanning tree of $G$.

\section{References}

[1] N. Alon, J. Balogh, B. Bollobás, and T. Szabó, Game domination number, Discrete Math. 256 (2002), 23-33.

[2] B. Brešar, S. Klavžar, and D. Rall, Domination game and an imagination strategy, SIAM J. Discrete Math. 24 (2010), 979-991.

[3] B. Brešar, S. Klavžar, and D. Rall, Domination game played on trees and spanning subgraphs, Discrete Math. 313 (2013), 915-923.

[4] M.M. Blank, An estimate of the external stability number of a graph without suspended vertices Prikl. Mat. i Programmirovanie 10 (1973), 3-11, 149.

[5] T. Dinski and X. Zhu, Game chromatic number of graphs, Discrete Math. 196 (1999), $109-115$.

[6] O. Favaron, H. Karami, R. Khoeilar, S.M. Sheikholeslami, and L. Volkmann, Proof of a conjecture on game domination, J. Graph Theory 64 (2010), 323-329.

[7] Z. Füredi, D. Reimer, and A. Seress, Hajnal's triangle-free game and extremal graph problems, Congr. Numer. 82 (1991), 123-128.

[8] M. Gardner, Mathematical games, Scientific American 244 (1981), 18-26. 
[9] T. W. Haynes, S. T. Hedetniemi, and P. J. Slater, Fundamentals of domination in graphs. Monographs and Textbooks in Pure and Applied Mathematics 208 (Marcel Dekker, 1998).

[10] H. A. Kierstead and A. V. Kostochka, Efficient graph packing via game colouring, Combin. Probab. Comput. 18 (2009), 765-774.

[11] H. A. Kierstead and T. Trotter, Planar graph coloring with an uncooperative partner, J. Graph Theory 18 (1994), 569-584.

[12] G. Košmrlj, Realizations of the game domination number, J. Combin. Opt. (to appear).

[13] U. Schauz, Mr. Paint and Mrs. Correct, Electron. J. Combin. 16 (2009), no. 1, Research Paper 77, 18 pp.

[14] R. Zamani, Hamiltonian Cycles through Specified Edges in Bipartite Graphs, Domination Game, and the Game of Revolutionaries and Spies, Ph.D. thesis, University of Illinois at Urbana-Champaign. Ann Arbor: ProQuest/UMI. (Publication No. AAT 3496787.)

[15] X. Zhu, Game coloring the Cartesian product of graphs, J. Graph Theory 59 (2008) 261-278.

[16] X. Zhu, On-line list colouring of graphs, Electron. J. Combin. 16 (2009), no. 1, Research Paper 127, 16 pp. 